\title{
Wachstumskern „Biologische Sensor-Aktor-Systeme auf der Basis von funktionalisierten Mikroorganismen" - BioSAM - Überblick
}

\author{
S. Johne ${ }^{1}$ \\ 1- Sprecher BioSAM-Molsurf GmbH \& Co. KG, Moritzburger Weg 67, 01109 Dresden/Deutschland \\ johne@molsurf.de
}

\section{Zusammenfassung}

Das zentrale Ziel des im Januar 2015 gegründeten Wachstumskerns „Biologische Sensor-AktorSysteme auf der Basis von funktionalisierten Mikroorganismen (BioSAM)" ist die Entwicklung einer neuartigen Generation von Ganzzellsensoren und -aktoren für technische Anwendungen, insbesondere für die Bioverfahrenstechnik, die Umwelttechnik und die Medizintechnik. Lebende Zellen sollen zur Bewertung der biologischen Wirkung von biologischen oder nichtbiologischen Analyten sowie zur Steuerung darauf beruhender Prozesse genutzt werden. Der innovative Ansatz beruht dabei auf der hohen Spezifität und Sensitivität biologischer Systeme.

Die angestrebte Lösung besteht darin, Mikroorganismen molekularbiologisch zu modifizieren und unter Einhaltung der gesetzlich vorgeschriebenen Sicherheitsstandards in technische Systeme zu integrieren. Zunächst stehen neue Produkte für Anwendungen in der Wasser- und Abwasserbehandlung, der Klimatechnik und der Biogasgewinnung im Vordergrund. Die Anwendungsfelder sollen mittelfristig weiter ausgedehnt werden, beispielsweise in Richtung Medizintechnik und Biotechnologie. Die Vermarktung der Produkte umfasst sowohl Einzelkomponenten, wie z. B. Sensoren, als auch Komplettsysteme.

Keywords: Ganzzellsensor, Wachstumskern, Sensor-Aktor-System,

\section{Ziele des Bündnisses}

Zwölf Unternehmen aus der Region Mitteldeutschland mit dem Zentrum Dresden widmen sich im Verbund mit zehn Arbeitsgruppen aus insgesamt sechs Forschungseinrichtungen dieser vielversprechenden Technologie. Die unternehmerische Vision des Bündnisses ist darauf gerichtet, Erkenntnisse aus der Spitzenforschung mit Hilfe eines starken unternehmerischen Netzwerkes zu einer Technologieplattform zusammenzufügen und zu kommerzialisieren. Das Bündnis wird im Rahmen der Innovationsinitiative „Unternehmen Region“ des Bundesministeriums für Bildung und Forschung (BMBF) gefördert und soll als Wachstumskern den Keim für die Entstehung eines starken regionalen Wirtschaftsclusters schaffen. Unter dem Leitmotiv "Lebende Zellen auf Oberflächen - hochempfindlich und trotzdem stabil" soll die BioSAM-Technologieplattform für eine breite wirtschaftliche Nutzung von zellbasierten Sensor-Aktor-Systemen in den
Bereichen Bioverfahrenstechnik, Wasser- und Umwelttechnologie sowie Klimatechnik erschlossen werden.

\section{Kernkompetenzen des Bündnisses}

- Funktionalisierung von Mikroorganismen zur Erfüllung kundenspezifischer Funktionen

- Immobilisierung lebender Mikroorganismen

- Multiparametrische Systemlösungen mit robusten elektronischen und optischen Schnittstellen für zellbasierte Sensor-AktorSysteme

Die Techniologieplattform setzt sich aus 5 Basiskomponenten zusammen:

1. Variable Funktionsanpassung durch gentechnische Modifizierung (Abb. 1)

2. Aktivitätserhaltende Immobilisierung von Zellen und Molekülen (Abb. 2)

3. Definierte Reaktionsräume von extremen Umgebungsparametern (Abb. 3) 
4. Schaltbare biologische Funktionsschichten (Abb. 4)

5. Robuste modulare Lösungen mit definierten biohybriden elektronischen Schnittstellen (Abb. 5)

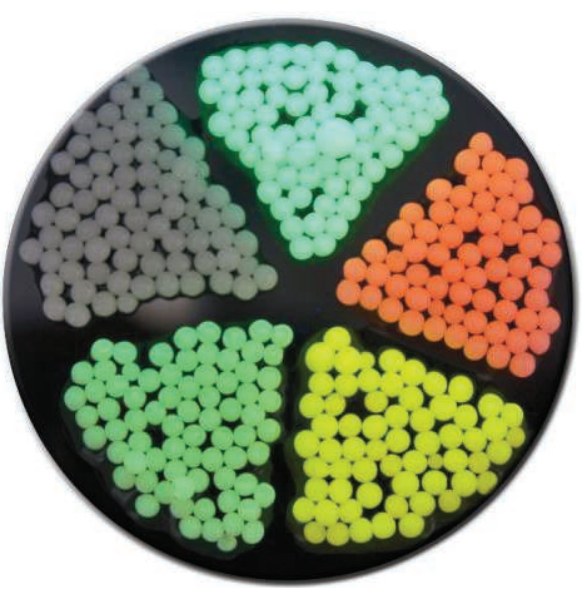

Abb. 1: In Alginatkugeln eingebettete molekularbiologisch modifizierte Bakterien, die verschiedene fluoreszierende Proteine bilden

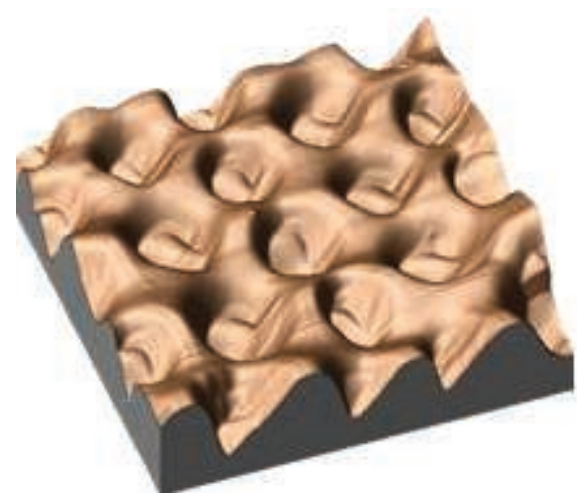

Abb. 2: Dreidimensionale Rekonstruktion einer AFM-Aufnahme von Sol-Gelimmobilisierten, hochdicht und geordnet abgelagerten Hefezellen

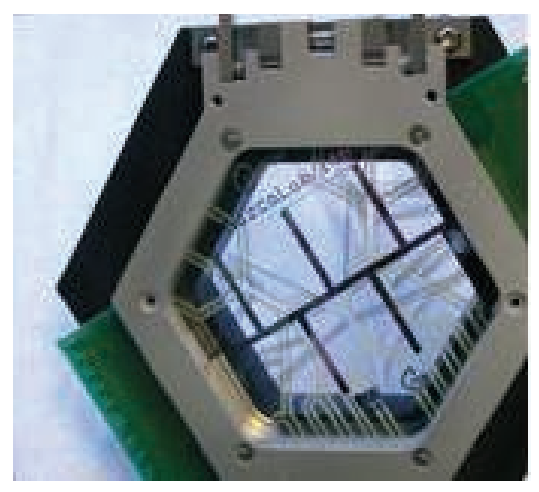

Abb. 3: Mikrofluidische Flusszelle auf Basis von Glas-Polymer-Glas

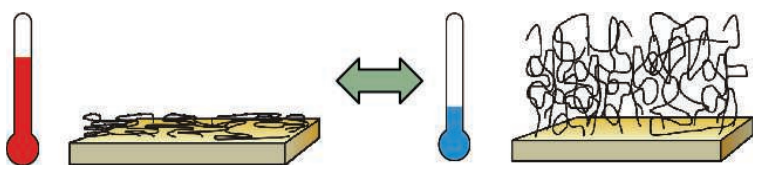

Abb. 4: Temperaturabhängige Reinigung der Sensoroberfläche in situ

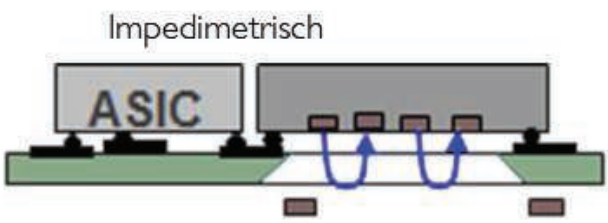

Optoelektronisch

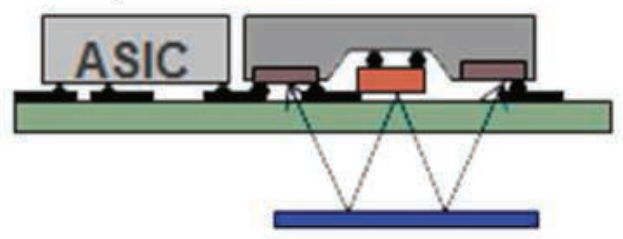

Abb. 5: Prinzipdarstellung von impedimetrischen und optolektronischen Sensoren

\section{Die Entwicklung der Plattform im Bündnis erfolgt durch 4 FuE Projekte:}

HIGS:

Ziel des Vorhabens HIGS „Hochintegrierte Ganzzellsensoren für die Umwelt- und Medizintechnik" ist die Entwicklung einer neuartigen Generation von Ganzzellsensoren für die Umwelt- und Medizintechnik, die durch die Response lebender Zellen die Bewertung der biologischen Wirkung von biomolekularen oder nichtbiologischen Analyten ermöglichen. Die angestrebte innovative neuartige Lösung soll es erlauben, dass zukünftig solche Sensoren, bei denen gentechnisch modifizierte Mikroorganismen zum Einsatz kommen, durch Integration in ein modulares Mikrofluidiksystem auch beim Arbeiten in konventioneller Arbeitsumgebung sowie in offenen technischen Systemen den Sicherheitsstandard S1 erfüllen. Dies soll durch zwei in das Mikrofluidiksystem integrierte Module für die Detektion von einzelnen möglicherweise freien Zellen und deren sichere Denaturierung sichergestellt werden. Zusätzlich zu der hohen Standzeit der Sensoren soll die Entwicklung erstmalig durch neuartige Konzepte zur internen Referenzierung mittels elektrischer Messung und zur biologisch-chemischen bzw. physi- 
kalischen Regenerierung der Sensoroberfläche applikationsreif erweitert werden.

\section{NAPYS:}

(Nanostrukturierte pyroelektrische und polymere Schichten für funktionell optimierte und robustere Biosensoren): Biosensorische Problemstellungen finden sich heutzutage überall in der industriellen Praxis, jedoch sind bislang nur wenige Lösungen hierzu kommerziell verfügbar. Die bislang nur unzureichend bearbeitete Problematik liegt in der Stabilität der Schnittstelle zwischen Biologie und Sensortechnik. Hierbei fehlen Lösungen, die es erlauben biologische Sensoren unter harschen Industriebedingungen auch über längere Zeiten einsetzen zu können. Das Verbundprojekt NAPYS verfolgt verschiedene Ansätze, hier für praktisch einsetzbare Konzepte zu entwickeln, die es ermöglichen robuste Biosensoren für den industriellen Einsatz zu entwickeln. Zu diesem Zweck werden verschiedene Arten der Beschichtung entwickelt, die eine Optimierung hinsichtlich Langzeitstabilität und Robustheit erlauben. Erprobt werden diese Verfahren in Kombination mit zu diesem Zweck optimierten impedimetrischen und optischen Sensoren. Außerdem werden elektronische Messmodule und kompakte, robuste Systeme für den dezentralen Einsatz der Biosensoren entwickelt. Die Konzepte, Sensoren und Messmodule sind Bestandteil des Plattformkonzeptes, das im Rahmen des Wachstumskerns BioSAM in mehreren Verbundprojekten entwickelt wird. Sie werden an verschiedenen Stellen mit den Ergebnissen der anderen Verbundprojekte kombiniert und koordiniert. Als Referenzanwendung für die praktische Erprobung dienen raumlufttechnische Anlagen in NAPYS, sowie Wasserbehandlung und Bioverfahrenstechnik aus den anderen Verbundprojekten des Wachstumskerns.

\section{BIOGAS}

(Autarke multiparametrische MonitoringSysteme für die Biogasproduktion zur Optimierung ihrer Leistungsparameter): Ziel der geplanten Forschungsarbeit ist ein inline arbeitendes Mess- und Auswertesystem für die Zustandsbeschreibung von biotechnologischen Prozessen. Als Referenzanwendung ist das Monitoring von Prozessbedingungen der Biogasherstellung vorgesehen. Mit diesem System sollen wichtige biochemische Prozessindikatoren spezifisch erfasst werden, wobei vorrangig in der Prozessflüssigkeit gelöste Spurenelemente, sowie bei der Referenzanwendung Essigsäure als wesentlicher Bestandteil der Prozessflüssigkeit bei der Hydrolyse anaerob vergorener Substrate im Fokus stehen. Aus Essigsäure wird auf direktem Weg durch methanogene Mikroorganismen Biogas erzeugt, während die Spurenelemente die Umsetzungseffizienz dieser Mikroorganismen maßgeblich beeinflussen. Als hoch sensitive Schnittstelle des zu entwickelnden Messsystems zu den biotechnologischen Prozessen dienen immobilisierte Zellen, mit denen die Konzentrationen der gewünschten Komponenten erfasst und über neu entwickelte Transducer (Sensorwandler) an ein Plattformspezifisches System zur Signalverarbeitung übergeben werden. Der Ansatz für die Referenzanwendung in Biogasanlagen sieht weiterhin die Kopplung von elektrochemischen sowie Gas- und Ganzzell-basierten Sensoren zu Multiparameter-Sensoren vor.

\section{BioNEWS:}

(Reaktive, regenerierbare Biohybridsysteme zum Nachweis und zur Entfernung von Wert- und Schadstoffen aus wässrigen Systemen): Das Ziel des Vorhabens besteht in der Ausnutzung der BioSAM- Technologieplattform zur Entwicklung von hochselektiven und langzeitstabilen biologischen Sensor-Aktor-Strukturen zum Zwecke einer nachhaltigen Wasser- und Ressourcennutzung. So sollen zum einen gering konzentrierte organische Schadstoffe (refraktäre Wasserinhaltsstoffe) oder anorganische Wertstoffe (Industriemetalle) über neuartige Sensoren detektiert und zum anderen verschiedene Aktor-Systeme nach dem gleichen Konzept entwickelt werden, die es ermöglichen, derartige Stoffe aus dem Wasser zu entfernen. Ziel ist es, organische Verunreinigungen (wie Pharmazeutika, BTEX, AOX) aus Reinst- oder Rohwässern zu eliminieren, was insbesondere auch für die Trinkwasseraufbereitung genutzt werden kann, oder strategisch wichtige Metalle (wie Indium, Germanium, Gallium, Seltene Erden) aus Bergbau- und industriellen Prozesswässern zurückzugewinnen. Die entsprechenden Lösungskonzepte zur Sensorik und zur Entfernung/ Gewinnung gering konzentrierter Wasserinhaltsstoffe basieren auf der Nutzung maßgeschneiderter gentechnisch modifizierter Zellen und natürlicher Isolate mit spezifischen zellulären Bindungsstrukturen. 


\section{Die Partner im Wachstumskern:}

DMOS $\mathrm{GmbH}$,

Bergstr. 4, 01069 Dresden, www.dmos2002.de

G.E.O.S. Ingenieursgesellschaft $\mathrm{mbH}$, Schwarze Kiefern 2, 09633 Halsbrücke, www.geosfreiberg.de

GeSiM Gesellschaft für Silizium-Mikrosysteme $\mathrm{mbH}$

Bautzner Landstr. 45, 01454

Großerkmannsdorf, www.gesim.de

GICON Großmann Ingenieur Consult GmbH, Tiergartenstr. 48, 01219 Dresden, www.gicon.de

IFU Diagnostic Systems $\mathrm{GmbH}$, Gottfried-Schenker-Str. 18, 09244 Lichtenau, www.ifu-diagnostic.de

IFU GmbH Privates Institut für Umweltanalysen,

Gottfried-Schenker-Str. 18, 09244 Lichtenau, www.ifu.de

MH Wassertechnologie $\mathrm{GmbH}$, Ringstr. 22, 01468 Boxdorf bei Dresden, www.mh-wassertechnologie.de

Molsurf $\mathrm{GmbH} \&$ Co. KG, Moritzburger Weg 67, 01109 Dresden, www.molsurf.de

Sciospec Scientific Instruments $\mathrm{GmbH}$, Grimmaische Str. 92, 04828 Bennewitz OT Pausitz, www.sciospec.de

UMEX GmbH Dresden, Moritzburger Weg 67, 01109 Dresden, www.umex.de

UV-EL GmbH \& Co. KG, Moritzburger Weg 67, 01109 Dresden, www.uv-el.de

CiS Forschungsinstitut für Mikrosensorik $\mathrm{GmbH}$,

Konrad-Zuse-Str. 14, 99099 Erfurt, www.cismst.de

GMBU e.V.

Dresden-Rossendorf, Bautzner Landstr. 45, 01454 Radeberg, www.gmbu.de
Helmholz-Zentrum Dresden-Rossendorf. e.V., Bautzner Landstr. 400, 01328 Dresden, www.hzdr.de

Kurt-Schwabe-Institut für Mess- und Sensortechnik e.V.

Kurt-Schwabe-Str. 4, 04736 Waldheim, www.ksi-meinsberg.de

Technische Universität Dresden, 01062 Dresden, www.tu-dresden.de Institut für Wasserchemie (IfWC) Institut für Werkstoffwissenschaft (IfWW) Institut für Genetik (IfG) Institut für Technik- und Umweltrecht (ITUR) Institut für Festkörperelektronik (IFE)

Universität Leipzig - Institut für Biochemie (IBC),

Brüderstr. 34, 04103 Leipzig, www.biochemie.uni-leipzig.de 